Ann. Zootech., I970, 19 (I), 67-73.

\title{
NIVEAU ÉNERGÉTIQUE, PROTÉIQUE ET FÉCONDITÉ DES VACHES LAITIERES. INFLUENCE D'UNE SUPPLÉMENTATION ALIMENTAIRE POST-GESTRALE
}

\author{
R. GIROU et M. BROCHART \\ Laboratoire de Physiopathologie de la Nutrition, \\ Centre de Recherches de Clermont-Ferrand, 63 - Saint-Genès-Champanelle \\ Institut national de la Recherche agronomique
}

RÉSUMÉ

Les apports énergétiques, mesurés en unités fourragères (UF), et protéiques, mesurés en grammes de matière azotée digestible (MAD), fournis à I zoo vaches de racc Montbéliarde soumises au contrôle laitier ont été mesurés. On n'a pas pu confirmer sur cette population l'influence négative, signalée par divers auteurs, du déficit ou de l'excès d'énergie sur la fécondité, mesurée par le taux de non-retour en chaleur trois mois après la première insémination artificielle. Toutefois, une supplémentation alimentaire post-cestrale, durant six jours, de $3 \mathrm{~kg}$ par jour d'un aliment à 0,9 UF par kg et $22 \mathrm{p}$. roo de matière protéique brute, administré à la moitié des vaches, tend à augmenter la fécondité des vaches carencées en énergie et à diminuer celle des vaches en recevant un excès (respectivement + II,5 p. roo et - 5, I p. Ioo par rapport aux vaches témoins ; $p<0,06)$.

Une tendance à l'abaissement de la fécondité lorsque les apports azotés sont excessifs a été observée sur la population de I 300 vaches ; le phénomène a été confirmé par l'analyse des observations effectuées sur un troupeau expérimental de 49 vaches, au cours de 3 années consécutives ; les apports d'énergie étaient ajustés chaque semaine aux besoins, les apports azotés étant variables qualitativement et quantitativement; le seuil d'excès de matières azotées digestibles compatible avec une fécondité normale se situait pour ce troupeau entre 200 et $300 \mathrm{~g}$ par jour $(p<0,0 \mathrm{r})$.

\section{INTRODUCTION}

L'existence d'une relation entre le niveau alimentaire et la fécondité des vaches est basée sur 2 groupes d'observations différentes. Dans le premier groupe, les auteurs, travaillant généralement sur de grands effectifs, ont utilisé des critères indirects et globaux du niveau nutritionnel, notamment la persistance de lactation (BROCHART, I966; KING, I968) et l'évolution pondérale après le vêlage (WILTiBANK, I964; 
MCCluRE, I965; KING, I968). Dans le deuxième groupe, des expériences d'alimentation contrôlée portant nécessairement sur des effectifs expérimentaux plus réduits ont mis en évidence que des régimes soit fortement carencés en énergie (WILTBANK, Ig63 ; BLAKELY, I963) soit nettement excédentaires (SAIDDUDIN et al., rg68) exerçaient, selon des mécanismes évidemment différents, une action négative sur la fécondité.

On peut se demander si les résultats des expériences du deuxième groupe, plus rigoureuses que celles du premier, ont néanmoins une signification générale dans les conditions habituelles d'exploitation d'un troupeau de vaches laitières; par ailleurs, l'absence d'informations sur l'influence du niveau azoté sur la fécondité est regrettable.

C'est pourquoi il nous a paru intéressant:

$I^{0}$ de vérifier sur un effectif assez important de I 300 vaches laitières issues de 400 troupeaux du département du Jura, la généralité de la relation entre fécondité et niveau énergétique, mesuré avec la meilleure précision compatible avec les conditions de l'expérience. On a considéré d'une part l'apport absolu d'énergie, d'autre part l'apport relatif assuré par le concentré, puisqu'il a été signalé que des apports élevés de concentré sont associés à une baisse de fécondité (FRANZOS, I968; K+LI et al., Ig68).

$2^{\circ}$ D'étudier sur ce même matériel animal l'influence des apports azotés. L,es résultats obtenus suggérant une influence sur la fécondité, nous avons ensuite procédé à une vérification sur un troupeau expérimental.

Parallèlement aux observations effectuées sur l'effectif de I 300 vaches, nous avons réalisé sur celui-ci une expérience consistant à donner, à la moitié des vaches, une supplémentation alimentaire sous forme d'un aliment concentré pour vaches laitières pendant six jours après l'insémination. Cette expérience avait pour but de voir si cette supplémentation pourrait améliorer la fécondité, soit par un effet compensateur d'une éventuelle carence, soit par un effet dynamique d'élévation du niveau alimentaire, un peu analogue à ce qui est pratiqué lors du "flushing » chez la Brebis.

Nous avons retenu le post-œstrus parce que cette période est chez la Ratte (BERG, 1965, I967) et la Brebis (EDEY, I966) particulièrement sensible à une variation de brève durée du niveau alimentaire ; or, chez la Vache, l'importance de la mortalité embryonnaire précoce $\left(5^{\circ} \mathrm{p}\right.$. Ioo des cas de retours en chaleurs selon GRADEN et al. I968) suggère que la période post-œestrale est également une période critique.

\section{MATÉRIEI, ET MÉTHODES}

L'expérience s'est déroulée dans le Jura, au cours de l'hiver i 968 -I969, en collaboration avec la Société " Jura-bétail n, dans 400 étables inscrites au contrôle laitier, ce qui présentait l'avantage d'avoir un cnsemble de données complet et séricux, mais aussi l'inconvénient de se trouver en présence d'asriculteurs d'une technicité nettement au-dessus de la moyenne ct d'animaux recevant une alimentation également supérieure à la moyenne, donc d'une moins grande variabilité des facteurs alimentaires.

L'expérience a porté sur 2 200 vaches, mais l'alimentation au moment de l'insémination a été relevéc pour I zoo vaches seulement. Eille était constituée essentiellement de foin de prairie naturclle, de céréales et de tourteau d'arachide. Les consommations ont été légèrement surestimées car il n'a pas été tenu compte des refus. Suivant une répartition au hasard, la moitié des vaches de chaque exploitation n'a pas reçu de supplément (Lot témoin) et l'autre moitié a reçu (Lot supplémenté) après l'insémination $3 \mathrm{~kg}$ par jour pendant six jours d'un aliment "vache laitière n courant, à 0,9 unité fourragère $/ \mathrm{kg}$ et $22 \mathrm{p}$. Ioo de matière protéique brute. 
L'étude complémentaire des variations de fécondité en fonction du niveau de consommation azotée a porté sur les relevés et observations effectués sur un effectif expérimental de 49 vaches laitières de la station de l'Élevage du Centre de Theix, au cours de 3 années consécutives. Les animaux recevaient une ration ajustée du point de vue énergétique chaque semaine à leur poids et à la production laitière, les apports azotés variant, par contre, de façon non contrôléc et étant généralement excédentaires. Les consommations étaient mesurées de manière précise, par pesée des quantités distribuées et des refus. Les analyses d'aliments étaient effectuées régulièrement.

Durant les hivers I 966-67 et I967-68, l'azote alimentaire provenait de sources variées, et notamment de tourteau d'arachide, et pendant 1'hiver I968-69, la source principale d'azote fut la Iuzerne déshydratée.

La fécondité a été mesuréc par le taux de non-retour en chalcurs trois mois après l'insé. mination artificielle.

\section{RÉSULTATS}

Pour l'analyse des résultats, la population de I 300 vaches a été répartie en 3 classes de niveau d'apport énergétique et protéique, à savoir niveau bas, moyen, élevé. Cette méthode ne permet donc qu'une analyse au niveau individuel, et néglige le facteur " exploitation ", pourtant décisif en matière d'infécondité ; le trop faible nombre de vaches ayant participé à l'expérience dans chaque exploitation ne nous a pas permis de faire une analyse à ce niveau.

\section{$1^{\circ}$ Apport d'énergie et fécondité Modification apportée par la supplémentation}

Chez les vaches témoins (tab1. I) on n'observe pas de relation significative entre niveau énergétique et fécondité. La supplémentation est sans action sur les vaches

TABLEAU I

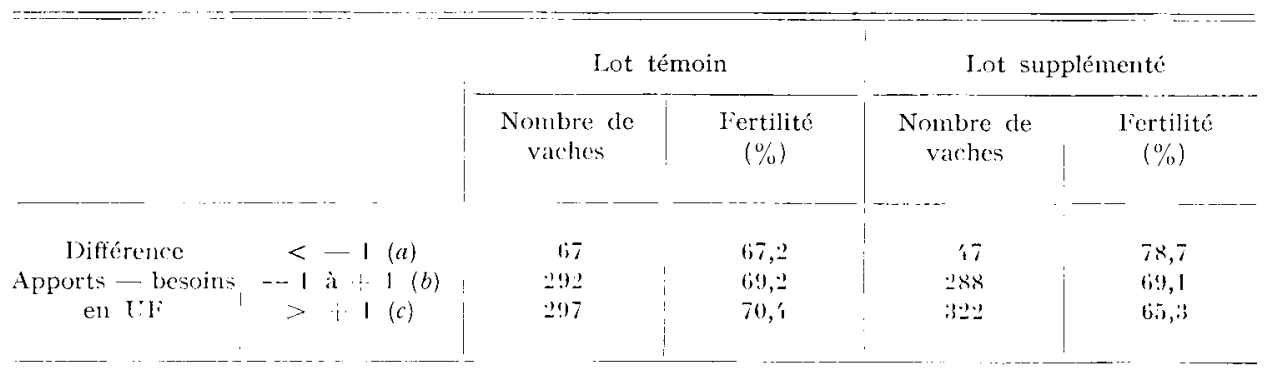

La différente induite par la supplémentation entre les groupes $(a)$ et $(c)$ est proche de la siguification $(p<0,0(i)$.

recevant des apports proches des besoins (à \pm I UF près) mais tend à augmenter fortement la fécondité des vaches carencées $(+$ I, $5 \mathrm{p}$. Ioo) et à diminuer celle des vaches recevant un excès d'énergie $(-5, \mathrm{I}$ p. Ioo). Cette inversion de l'effet de la supplémentation est très proche du seuil de signification $(p<0,06)$.

L'augmentation de l'apport relatif d'énergie par le concentré est, chez les vaches témoins, associée à une baisse de fécondité (tabl. 2). La supplémentation renforce cette tendance (différence de 7,4 p. Ioo entre les classes extrêmes), sans que l'effet soit cependant significatif. 
TABLEAU 2

\begin{tabular}{|c|c|c|c|c|c|}
\hline & & \multicolumn{2}{|c|}{ Lot témoin } & \multicolumn{2}{|c|}{ Lot supplémenté } \\
\hline & & $\begin{array}{c}\text { Nombre de } \\
\text { vaches }\end{array}$ & $\begin{array}{l}\text { Fertilité } \\
(\%)\end{array}$ & $\begin{array}{c}\text { Nombre de } \\
\text { vaches }\end{array}$ & $\begin{array}{c}\text { Fertilité } \\
(\%)\end{array}$ \\
\hline (\%) UF concentré & $<35$ & 151 & 72,7 & 139 & 72,7 \\
\hline $\mathrm{UF}$ aliments & $35-55$ & 225 & 68,9 & 228 & 68,0 \\
\hline grossiers & $>55$ & 213 & 68,1 & $2 / 12$ & 65,3 \\
\hline
\end{tabular}

TABLEAU 3

\begin{tabular}{|c|c|c|c|c|c|}
\hline & & \multicolumn{2}{|c|}{ Lot témoin } & \multicolumn{2}{|c|}{ Lot supplémenté } \\
\hline & & $\begin{array}{c}\text { Nombre de } \\
\text { vaches }\end{array}$ & $\begin{array}{c}\text { Fertilité } \\
(\%)\end{array}$ & $\begin{array}{c}\text { Nombre de } \\
\text { vaches }\end{array}$ & $\begin{array}{c}\text { Fertilité } \\
(\%)\end{array}$ \\
\hline $\begin{array}{l}\text { Différence } \\
\text { Apports - besoins } \\
\text { en } g \text { MAD }\end{array}$ & $\begin{array}{c}<-150 \\
-150 \text { à }+150 \\
>+150\end{array}$ & $\begin{array}{l}129 \\
255 \\
220\end{array}$ & $\begin{array}{ll}72,1 & (1) \\
71,8 & (2) \\
64,5 & (3)\end{array}$ & $\begin{array}{l}109 \\
285 \\
216\end{array}$ & $\begin{array}{l}73,4 \quad(4) \\
69,8(5) \\
67,6 \quad(6)\end{array}$ \\
\hline
\end{tabular}

Différence $(1+4)-(3+6)$ proche de la signification $(p<0,06)$.

\section{TABLEAU 4}

\begin{tabular}{|c|c|c|c|c|}
\hline \multicolumn{2}{|c|}{$\begin{array}{l}\text { Différence apports - besoins par jour en } \\
\text { moyenne sur l'intervalle : } 18 \text { jours avant } \\
\text { et } 18 \text { jours après l'insémination }\end{array}$} & $\begin{array}{l}\text { Vaches ayant } \\
\text { nécessité } \\
\text { une seule } \\
\text { insémination }\end{array}$ & $\begin{array}{l}\text { Vaches ayant } \\
\text { nécessité } \\
\text { plusieurs } \\
\text { inséminations }\end{array}$ & $\begin{array}{l}\text { P. } 100 \text { de réussite } \\
\text { en première } \\
\text { insémination }\end{array}$ \\
\hline \multirow{3}{*}{ Hiver $68-69$} & $<+200 \mathrm{~g}$ MAD (1) & 7 & 3 & 70 \\
\hline & +200 à $+400 \mathrm{~g}$ MAD & 3 & 6 & 33 \\
\hline & $>+400 \mathrm{~g} \mathrm{MAD}(2)$ & 7 & 23 & 23 \\
\hline \multirow{2}{*}{ Hiver $67-68$} & $<+300 \mathrm{~g} \operatorname{MAD}(3)$ & 13 & 10 & 58 \\
\hline & $>+300 \mathrm{~g} \operatorname{MAD}(4)$ & 1 & 12 & 8 \\
\hline \multirow[t]{2}{*}{ Hiver $66-67$} & $<+300 \mathrm{~g}$ MAD & 13 & 9 & 59 \\
\hline & $>+300 \mathrm{~g} \mathrm{MAD}$ & 3 & 4 & 43 \\
\hline
\end{tabular}

Les groupes (1) et (2); (3) et (4) respectivement présentent des différences hautement significatives $(p<0,01)$. 


\section{$2^{\circ}$ Apport azoté et fécondité. Modification apportée par la supplémentation}

On observe (tab1. 3) chez les vaches témoins une tendance à la baisse de fécondité pour un excès d'apport supérieur à I50 g (écart de 7,6 p. I00 entre les classes extrêmes). La supplémentation ne modifie pas cette tendance. La différence de fécondité des vaches des deux classes extrêmes d'apport (témoins et supplémentées prises ensemble) est proche de la signification $(p<0,06)$.

L'analyse des résultats fournis par le troupeau expérimental (tabl. 4) met en évidence des différences hautement significatives $(p<0,0 r)$ de la fécondité en fonction des apports azotés, le setil de l'excès d'azote compatible avec une bonne fécondité se situant entre 200 et $300 \mathrm{~g} \mathrm{MAD} / \mathrm{jour}$.

\section{DISCUSSION}

L'absence de relation entre niveau énergétique et fécondité des vaches témoins ne nous permet pas cependant d'infirmer les résultats significatifs obtenus par d'autres auteurs parce que :

Io le matériel animal ne s'est pas révélé très favorable, le nombre de vaches fortement carencées étant faible ;

$2^{0}$ les tendances révélées par la supplémentation sont conformes aux données bibliographiques, à savoir amélioration de la fécondité chez les vaches carencées, et diminution chez les vaches recevant un excès d'énergie.

En ce qui concerne l'influence du taux d'énergie apporté par le concentré, nos résultats vont dans le même sens que ceux signalés par les auteurs ayant étudié ce problème. Le fait que le seuil de signification n'ait pas été atteint dans l'un et l'autre cas tient peut-être à ce que l'influence de ces facteurs a été masquée par celle des nombreux autres facteurs agissant sur la fécondité d'une population de $\mathrm{r} 300$ vaches.

L'influence de l'excès azoté, vraisemblable mais non significative $(p<0,06)$ sur la population de I 300 vaches, a été confirmée par l'observation sur un effectif expérimental plus réduit. On ne saurait donc $a$ priori dénier tout intérêt à l'analyse des facteurs pouvant agir sur la fécondité d'une population d'animaux présentant une grande variabilité, mais la preuve décisive de la réalité de l'action d'un facteur isolé dans ces conditions nécessite le plus souvent une expérimentation ou une observation plus rigoureuse sur un effectif limité et bien contrôlé. En ce qui concerne l'excès azoté, en particulier, cette méthode d'investigation s'est avérée fructueuse; nous n'avons trouvé en effet aucune référence se rapportant à notre observation, les auteurs ne s'étant intéressé jusqu'à présent qu'à l'influence de la carence azotée, et chez d'autres espèces que les bovins. Or, l'excès azoté paraît être un risque majeur de l'intensification de la production laitière.

La supplémentation au cours de la période post-œstrale parait avoir un effet très favorable chez les vaches carencées, le taux de fécondité atteignant près de 79 p. roo contre $67 \mathrm{p}$. Ioo chez les vaches témoins, alors qu'elle diminue la fertilité des vaches recevant un excès d'énergie (respectivement 70,4 et 65,3 p. I00) ; ce phénomène d'inversion des effets de la supplémentation, conforme à ce que l'on sait de l'influence du niveau énergétique sur la fécondité, et l'existence d'un seuil très proche 
de la signification $(p<0,06)$, nous paraissent constituer un argument en faveur de la réalité du phénomène déclenché par la supplémentation. Celle-ci est effectivement susceptible d'avoir une action physiologique, ce que nous avons démontré dans une autre expérience.

Nous avons obtenu (GIROU et BRochaR'T, I970) une action positive hautement significative de cette même supplémentation sur le déclenchement des chaleurs chez des vaches en ancstrus post-partum ( + I2,6 p. roo par rapport aux vaches témoins ; $p<0,02)$.

Il est classiquement admis que l'élévation du niveau nutritionnel entraîne une réponse de 1'axe hypothalamo-hypophysaire gonadotrope (LAMMING, I966), et un tel mécanisme est probablement impliqué dans l'action de la supplémentation, chez les vaches carencées en énergie ; il est concevable qu'intervenant au post-œstrus, la supplémentation puisse favoriser la libération de l'hormone lutéinisante $\mathrm{LH}$, le développement et l'activité du corps jaune, et, par suite, les premières étapes du développement embryonnaire.

En conclusion, si nous ne pouvons confirmer l'influence du niveau énergétique sur la fécondité, cette dernière est abaissée par un excès d'azote. La supplémentation post-œstrale de brève durée paraît susceptible d'améliorer la fécondité des vaches carencées en énergie.

$$
\text { Reçu pour publication en février } 1970 .
$$

\section{REMERCIEMENTS}

Nous tenons à remercier le directeur et le personnel de la Société "Jura-bétail " pour la contribution efficace qu'ils ont apportée à la réalisation de cette expérience.

Nous tenons également à remercier le groupe de recherches sur la production laitjère du Centre de Theix pour la contribution à la fourniture de données concernant le troupeau expéri. mental de vaches laitières.

\section{SUMMARY}

\section{ENERGY AND PROTEIN LEVEL OF 'THE DIET WI'TH REFERENCE TO FERTILITY IN THE DAIRY COW EFFECT OF A POST-CESTRUAL FEED SUPPLENENTATION}

The encrgy supply, measured in feed units (li. U.), and the protein supply, measured in grammes, of digestible nitrogenous matter (DNM) given to I 300 cows of the breed Pie-rouge de l'Est wcre recorded. The negative effect reported by different authors of deficiency or excess of energy upon fertility measured by the non-return rate three months after the first insemination, could not be confirmed during this trial. However, a post-œstrual feed supplementation during six days of $3 \mathrm{~kg}$ per day of a feedstuff containing $0,9 \mathrm{~F}$. U. per kgand $22 \mathrm{p}$. I oocrude protein applicd to half of the cows, tends to increase the fertility of the energy deficient cows and to decrease that of the cows receiving an excess of the supplement (respectively + I I, 5 p. Ioo and - 5, I p. Ioo compared with the controls ; $p<0.06)$.

In this population of I 3 oo cows, we observed a trend of decrcasing fertility when the protein supply was excessive; the phenomenon was confirmed when analyzing the observations made in an experimental herd of 49 cows during three consecutive years. The energy supply was adapted to the requirements each week, the protein supply being qualitatively and quantitatively variable.

In this herd, the treshold of excess of digestible nitrogenous matters compatible with normal fertility was situated between 200 and $300 \mathrm{~g}$ per day $(p<0,0 \mathrm{I})$. 


\section{RÉFÉRENCES BIBLIOGRAPHIQUES}

BERG B. N., 1965. Dietary restriction and reproduction in the rat. J. Nutr., 87, 344-348.

BERG B. N., I967. Maintenance of pregnancy in protein-deprived rats by transitory protein supplements during early gestation. J. Nutr., 92,66-70.

Blakely J. E., Thompson G. B., Brooks J. R., Stufflebeam C. E., Lasley J. 1F., ig63. Effects of nutrition on reproduction and physiology in heifers. J. Anim. Sci., 22, I 136.

Brochart M., 1966. Fertilité des vaches laitières et persistance de lactation. C. R. Acad. Agric., 52, 9I 5 -9I9.

EDEY T. X., I966. Nutritional stress and preimplantation embryonnic mortality in Merino sheep. J. Agric. Sci., 67, 287-293.

Franzos G., r968. The relationship between the milk fat percentage and fertility in dairy herds. Refuah Vet., 25, 28-32.

Girou R., Brochart M., 1970. Effet d'une supplémentation alimentaire de brève durée sur la venue en chaleur de vaches en anœstrus post-partum. Ann. Zootech., 19 (I), 75-77.

Graden A. P., Durward O. L. S., Mochow C. R., Mutter L. R., Ig68. Causes of fertilization failure in repeat breeding cattle. J. Dairy Sci., 51, 778-78 I.

Lamming G. E., I966. Nutrition and the endocrine system. Nutr. Abstr. Rev., 36, I-I3.

Saiduddix S., Riesen J. W., Tyler W. J., Casida L. E., I967. First estrus and ovulation in the post-partum dairy cow. J. Anim. Sci., 26, 1494 .

Tyrell H. F., Trimberger G. W., Morrow D. A., Merrill W. G., Reid J. T., Loosli J. K., I968. Liberal grain feeding of dairy cows. Proceedings 1968, Cornell Conference in feed manuf., oct $29^{-}$ 30-3I, 95-IO2.

Wiltbank J. N., Rowden W. W., Ingalls J. E., Zimmerman P. R., rg64. Influence of post-partum energy level on reproductive performance of Hereford cows restricted in energy intake prior to calving. J. Anim. Sci., 23, 1049-I053. 